\title{
The white dwarf population of tri-axial haloes
}

\author{
Santiago Torres ${ }^{*, \dagger}$, Judit Camacho ${ }^{*, \dagger}$ and Enrique García-Berro ${ }^{*, \dagger}$ \\ * Departament de Física Aplicada, Universitat Politècnica de Catalunya, c/Esteve Terrades 5, \\ 08860 Castelldefels, Spain \\ $\dagger$ Institut d'Estudis Espacials de Catalunya, c/Gran Capità s/n, 08034 Barcelona, Spain
}

\begin{abstract}
The nature of the several microlensing events observed by the MACHO team towards the Large Magellanic Cloud (LMC) is still a controversial subject. Substellar objects and stars with masses larger than $\sim 1 M_{\odot}$ have been ruled out as major components of a Massive Astrophysical Halo Object (MACHO) Galactic halo. Stars of near half-solar mass, in particular white dwarfs, appear to be the best candidates to explain the observed microlensing events. On the other hand, observational evidence based on the structure of the debris of the Sagittarius dwarf galaxy suggest that the Galactic halo is non-spherical. We use a tri-axial halo model and advanced Monte Carlo techniques to study the contribution of the halo white dwarf population to the dark matter content of the Galaxy.
\end{abstract}

Keywords: stars: white dwarfs - stars: luminosity function, mass function - Galaxy: stellar content - Galaxy: structure - Galaxy: halo — dark matter

PACS: 97.20.Rp, 97.10.Xq, 97.10.Yp, 98.35.Gi, 98.35.Ln, 95.35.+d

\section{INTRODUCTION}

Current cold dark matter theories predict axis-symmetric haloes which diverge from the canonical symmetric isothermal sphere. Moreover, current observations, such as the structure of comoving groups of stars in our Galaxy, or the microlensing experiments towards the Large Magellanic Cloud and the bulge of our Galaxy, support this possibility. Here we explore if such tri-axial haloes can explain the results of the MACHO team [1].

\section{THE MODEL}

We have modified our Monte Carlo simulator of the white dwarf and red dwarf populations of the Galactic halo [2,3] to include a tri-axial halo. We have assumed that the halo was formed $14 \mathrm{Gyr}$ ago in intense burst of star formation of duration $1 \mathrm{Gyr}$. We have adopted a Salpeter-like initial mass function. The velocities were randomly drawn according normal distributions and adopting a rotation velocity of $250 \mathrm{~km} / \mathrm{s}$. We considered red dwarfs to have masses in the range of $0.08<M / M_{\odot}<1$. For these stars up-to-date evolutionary models [4] have been adopted. The cooling sequences adopted in this work depend on the internal composition of white dwarfs. White dwarfs with masses smaller than $M_{\mathrm{WD}}=1.1 M_{\odot}$ have carbon-oxygen cores and, consequently, if they belong to the DA spectral class we adopt the most reliable cooling tracks available now [5]. If, on the contrary, the synthetic white dwarf has a hydrogen-deficient atmosphere we use cooling sequences appropriate for DB white dwarfs [6]. White dwarfs with masses larger than $M_{\mathrm{WD}}=1.1 M_{\odot}$ most probably have ONe cores, and for them we 


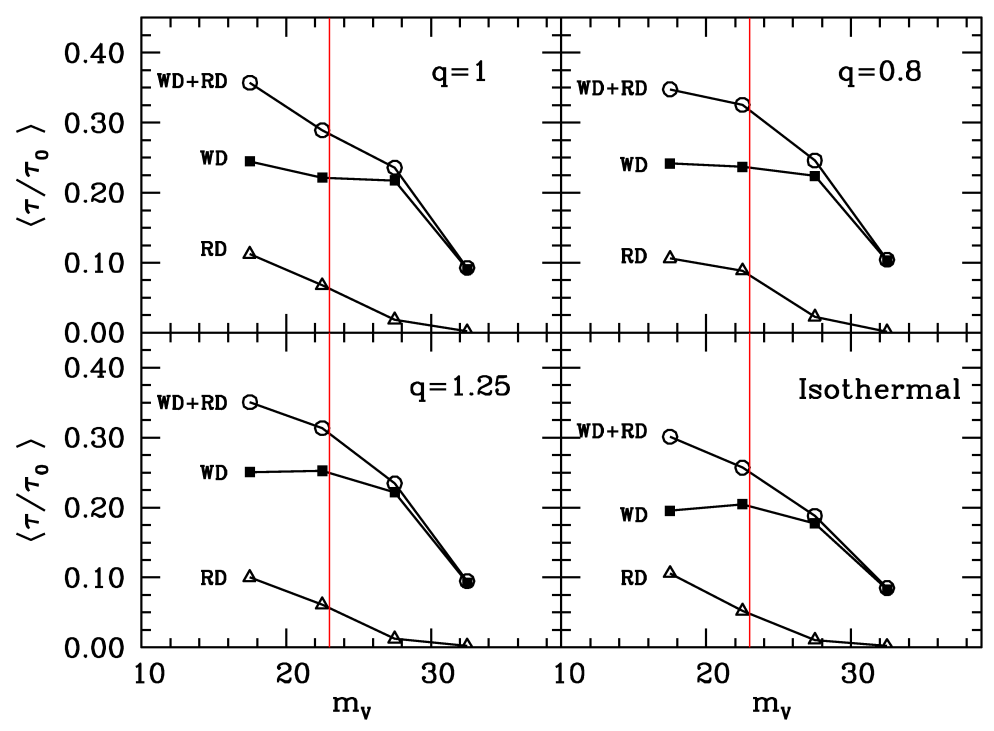

FIGURE 1. Microlensing optical depth towards the LMC as a function of the limiting magnitude. Solid squares and open triangles represent the white dwarf and red dwarf population, respectively, while the entire population is shown by open circles.

adopt the most recent cooling sequences available so far [7]. All these cooling sequences incorporate the most accurate physical prescriptions for the stellar interior.

For the spatial distribution of objects we have adopted a logarithmic dark halo potential [8],

$$
\Phi_{\mathrm{HALO}}=\frac{1}{2} v_{0}^{2} \ln \left(R^{2}+z^{2} / q^{2}+d^{2}\right)
$$

being the corresponding density distribution:

$$
\rho_{\text {HALO }}(R, z)=\left(\frac{v_{0}^{2}}{4 \pi G q^{2}}\right) \frac{\left(2 q^{2}+1\right) d^{2}+R^{2}+\left(2-q^{-2}\right) z^{2}}{\left(d^{2}+R^{2}+z^{2} q^{-2}\right)^{2}}
$$

where we have adopted $d=12 \mathrm{kpc}$ and $v_{0}=131.5 \mathrm{~km} / \mathrm{s}$, which gives a circular velocity of the Sun of $229 \mathrm{~km} / \mathrm{s}$. The parameter $q$ is allowed to vary from 0.8 to 1.25 , that is from oblate to prolate configurations.

Finally, in order to produce a set of microlensing events due to the white dwarf population we have generated a pencil towards the LMC. This pencil has been normalized to the local density of halo white dwarfs [9]. We have also simulated the population of stars of the LMC [10]. In our simulations we have considered a white dwarf to be responsable of a microlensing event if the angular distance between the white dwarf and the monitored star is smaller than the Einstein radius. Finally, we computed all the relevant observables, like the optical depth and the Einstein cross time. 
TABLE 1. Summary of results obtained for the entire population of microlenses towards the LMC for a realistic magnitude cut of $m_{v}>23^{\mathrm{mag}}$.

\begin{tabular}{lcccc}
\hline & & Tri-axial haloes & & Isothermal sphere \\
& $q=0.8$ & $q=1.0$ & $q=1.25$ & \\
\hline$\left\langle N_{\mathrm{WD}}\right\rangle$ & $0 \pm 1$ & $0 \pm 1$ & $0 \pm 1$ & $0 \pm 1$ \\
$\left\langle N_{\mathrm{RD}}\right\rangle$ & $0 \pm 1$ & $0 \pm 1$ & $0 \pm 1$ & $0 \pm 1$ \\
$\left\langle m_{\mathrm{WD}}\right\rangle\left(M / M_{\odot}\right)$ & 0.601 & 0.599 & 0.601 & 0.602 \\
$\left\langle m_{\mathrm{RD}}\right\rangle\left(M / M_{\odot}\right)$ & 0.210 & 0.242 & 0.259 & 0.227 \\
$\langle\mu\rangle\left({ }^{\prime \prime} \mathrm{yr}^{-1}\right)$ & 0.018 & 0.012 & 0.015 & 0.016 \\
$\langle d\rangle(\mathrm{kpc})$ & 2.93 & 3.99 & 3.88 & 3.28 \\
$\left\langle V_{\mathrm{tan}}\right\rangle\left(\mathrm{km} \mathrm{s}^{-1}\right)$ & 248 & 231 & 267 & 244 \\
$\left\langle\hat{t}_{\mathrm{E}}\right\rangle(\mathrm{d})$ & 41.6 & 60.0 & 46.3 & 45.3 \\
$\left\langle\tau / \tau_{0}\right\rangle$ & 0.326 & 0.289 & 0.314 & 0.257 \\
\hline
\end{tabular}

\section{RESULTS}

For each one of the different models detailed above we have performed a set of $10^{3}$ independent realizations, for which the average of any observational quantity and its corresponding standard deviation were computed. In Figure 1 we show the microlensing optical depth towards the LMC obtained in our simulations normalized to the observational value derived by the MACHO team, $\tau_{0}=1.2 \times 10^{-7}[1]$, as a function of the magnitude cut below which white dwarfs should be detected. Halo models with a logarithmic potential have optical depths slightly larger than those obtained using a isothermal sphere. For a realistic magnitude cut, $m_{v}>23^{\mathrm{mag}}$ — represented as a vertical thin line — the highest contribution is obtained for an oblate halo model with $q=0.8$. Also, the contribution of the white dwarf population doubles that of the red dwarf population. Nevertheless, our Monte Carlo simulations provide additional information. We summarize it in Table 1, where we show the results towards the LMC for the different halo models. In particular, we show the number of microlensing events and the average mass of the microlenses for the white dwarf and red dwarf population, the average proper motion, the distance, and the average tangential velocity of the microlenses, the corresponding Einstein crossing time and the contribution to the microlensing optical depth. None of these halo models is able to produce the 11 microlensing observed events observed by the MACHO collaboration [1]. Finally, in Figure 2 we show the different estimates of the halo mass fraction $f$, as a function of the lens mass. The $95 \%$ confidence level contours of the MACHO, EROS [11] and OGLE-II [12] experiments are shown as solid lines. It is remarkable that a value of $f=0.08$, obtained for the oblate halo model, agrees with all the observational results.

\section{CONCLUSIONS}

We have analyzed the contribution of white dwarfs and red dwarfs to the microlensing optical depth towards the LMC for different logarithmic halo potentials (ranging from oblate to prolate models). Our results show that for a realistic magnitude cut, $m_{v}>23^{\mathrm{mag}}$, the highest contribution is obtained for an oblate halo. For this model the optical depth 


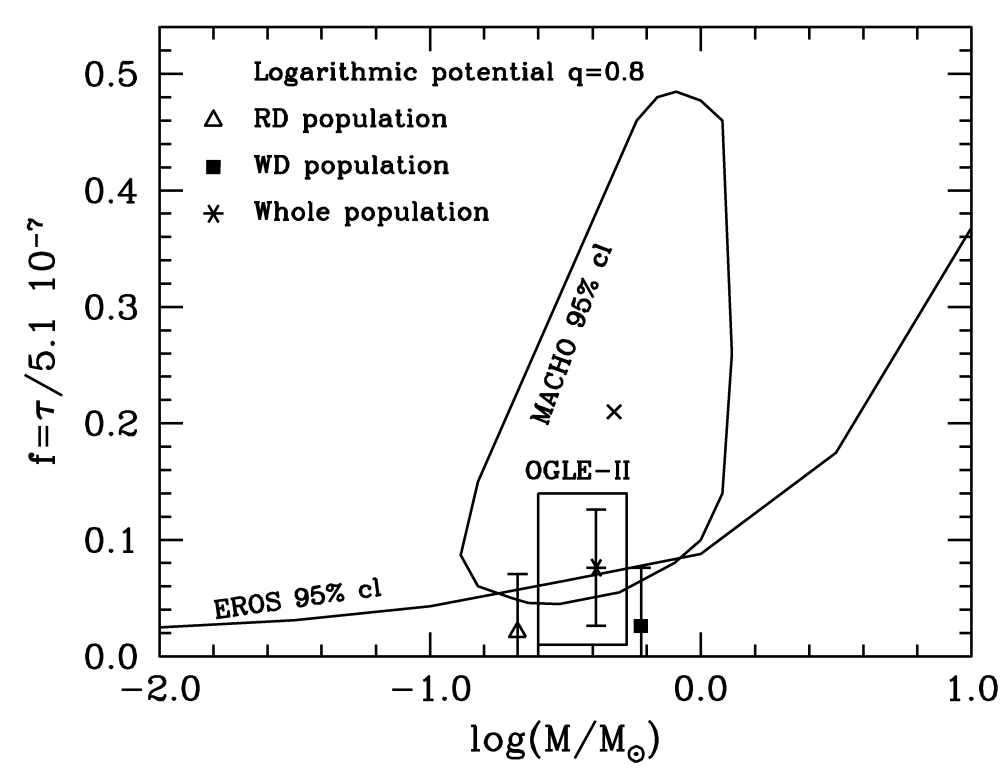

FIGURE 2. Halo dark-matter fraction as a function of the mass lens.

is $\sim 25 \%$ larger than that of the isothermal halo model. We have also found that for this model the fraction of dark matter locked in the form of MACHOs increases moderately, reaching $f=0.08$. We also find that our simulations are fully consistent with observations.

\section{ACKNOWLEDGMENTS}

This work was supported by MEC grant AYA08-04211-C02-01, and by the AGAUR.

\section{REFERENCES}

1. C. Alcock et al., Astrophys. J. 542, 281-307 (2000).

2. S. Torres, J. Camacho, J. Isern, and E. García-Berro, Astron. \& Astrophys. 486, 427-435 (2008).

3. S. Torres, J. Camacho, J. Isern, and E. García-Berro, Astron. \& Astrophys. 511, A88 (2010).

4. I. Baraffe, G. Chabrier, F. Allard, and P. H. Hauschildt, Astron. \& Astrophys. 337, 403-412 (1998).

5. M. Salaris, E. García-Berro, M. Hernanz, J. Isern, and D. Saumon, Astrophys. J. 544, 1036-1043 (2000).

6. O. G. Benvenuto, and L. G. Althaus, Month. Not. Roy. Astron. Soc. 288, 1004-1014 (1997).

7. L. G. Althaus, E. García-Berro, J. Isern, A. H. Córsico, and R. D. Rohrmann, Astron. \& Astrophys 465, 249-255 (2007).

8. A. Helmi, Month. Not. Roy. Astron. Soc. 351, 643-648 (2004).

9. S. Torres, E. García-Berro, and J. Isern, Astrophys. J. Lett. 508, L71-L74 (1998).

10. G. Gyuk, N. Dalal, and K. Griest, Astrophys. J. 535, 90-103 (2000).

11. P. Tisserand et al., Astron. \& Astrophys. 469, 387-404 (2007).

12. Ł. Wyrzykowski et al., Month. Not. Roy. Astron. Soc. 397, 1228-1242 (2009). 